Musées, Patrimoine et Culture scientifiques et techniques

\title{
PLUG : les secrets du musée
}

Recherche d'une médiation entre virtualité et réalité

Isabelle Astic et Coline Aunis

\section{(2) OpenEdition}

Édition électronique

URL : http://journals.openedition.org/ocim/240

DOI : 10.4000/ocim.240

ISSN : $2108-646 \mathrm{X}$

Éditeur

OCIM

\section{Édition imprimée}

Date de publication : 1 septembre 2009

Pagination : 5-11

ISSN : 0994-1908

\section{Référence électronique}

Isabelle Astic et Coline Aunis, «PLUG : les secrets du musée », La Lettre de l'OCIM [En ligne], 125 | 2009, mis en ligne le 01 septembre 2011, consulté le 10 mai 2019. URL : http://journals.openedition.org/ ocim/240 ; DOI : 10.4000/ocim.240 


\section{PLUG : les secrets du musée}

\section{Recherche d'une médiation entre virtualité et réalité}

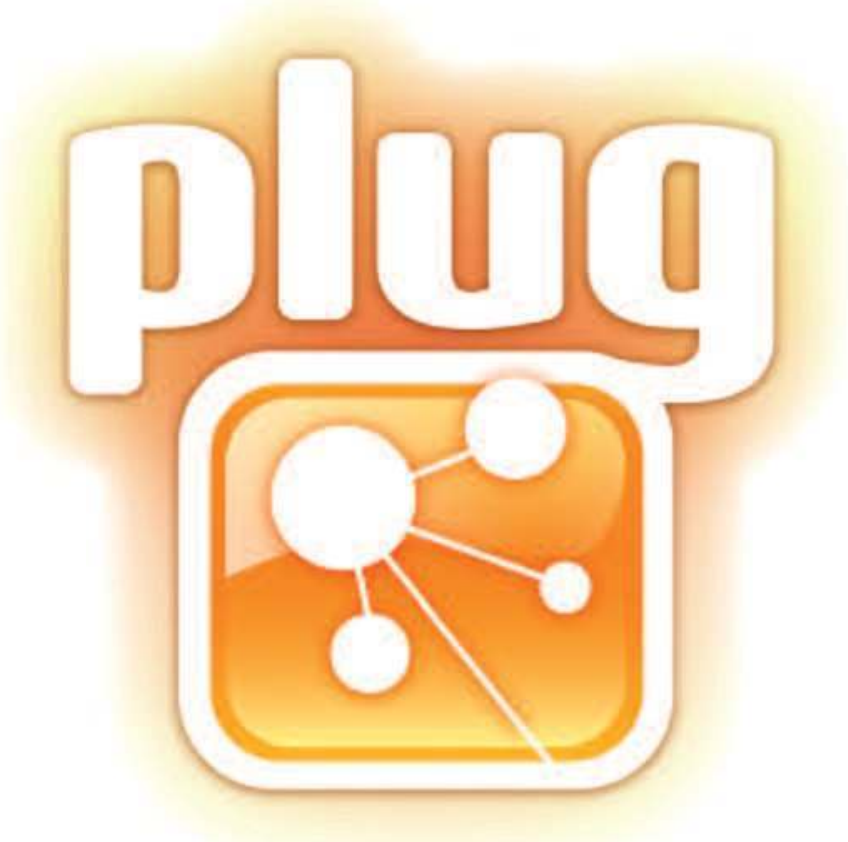

Logo du projet PLUG (Play Ubiquitous Game and Play more) ๑) PLUG

* Isabelle Astic est chargée de collection Informatique, Télécommunications et Réseaux au musée des Arts et Métiers isabelle.astic@cnam.fr Coline Aunis est chargée de projets Web et Multimédia au musée des Arts et Métiers coline.aunis@cnam.fr
Isabelle Astic et Coline Aunis *

Destiné à compléter l'offre de visite traditionnelle du musée des Arts et Métiers, en rendant le visiteur plus actif, le jeu «PLUG : les secrets du musée » a été conçu comme un nouveau mode original de médiation : les responsables du projet mettent en lumière les spécificités de l'architecture et des règles du jeu ainsi que les différentes étapes de sa mise au point et de sa réalisation.

Depuis janvier 2008, le musée des Arts et Métiers participe au projet PLUG (Play Ubiquitous Game... and play more), en partie financé par l'ANR (Agence Nationale de la Recherche) et labellisé par le pôle de compétitivité CAP DIGITAL (1). Il s'agit d'un projet de recherche industrielle sur 24 mois dont le but est de proposer une autre façon de vivre une visite, sous la forme d'un jeu pervasif ou ubiquitaire. Un monde pervasif est un monde où les objets communiquent avec l'homme et entre eux. Par exemple, un réfrigérateur qui détecterait la sortie du dernier yaourt et mettrait automatiquement à jour la liste des courses participe à ce monde pervasif. Un jeu pervasif connecte donc le joueur aux objets qui l'entourent et les objets entre eux. Dans le cadre d'un musée, cette interaction permet d'associer à chaque objet un contenu pédagogique. Elle doit induire un comportement actif du joueur et lui permettre d'aller vers l'information, tout en valorisant des objets des collections. Mais PLUG s'attache aussi à évaluer l'acceptabilité des solutions proposées tant du point de vue socio-culturel, qu'économique et industriel. Cette 
validation s'effectue chaque année et à l'occasion de la Fête de la Science. C'est pourquoi, PLUG réunit des partenaires académiques et industriels d'horizons différents : le musée des Arts et Métiers, le laboratoire de recherche en informatique CNAM-CEDRIC (systèmes embarqués mobiles, jeux vidéo et informatique émotionnelle), l'Institut Télécom Management (sociologie) \& Sud Paris (intergiciels), l'Institut Paris Tech (sciences de la communication et design d'environnement), le laboratoire L3i de l'université La Rochelle (jeux et interaction), Orange-France Télécom (opérateur de téléphonie mobile), Net Innovations (intégrateur de logiciels sur systèmes embarqués et mobiles), TetraEdge (studio de développement de jeux) auxquels s'associe une association de jeux grandeur nature : DUNE. Les compétences des partenaires vont des sciences humaines aux sciences et technologies de la communication et permettent ainsi de répondre à toutes les questions que pose un tel jeu.

Dans ce projet, le rôle du musée des Arts et Métiers est multiple. Il participe à la définition de l'architecture de ce jeu et à ses règles. Cependant, le musée doit aussi veiller à ce que ce jeu ne soit pas qu'un simple jeu dans l'enceinte de l'établissement, mais surtout un véhicule pédagogique, un " serious game ». Cette dimension est essentielle. Le musée organise aussi et déploie les nombreuses sessions de tests de validation du prototype, ainsi que l'expérimentation « grandeur nature ». Une première version du jeu a été développée durant l'année 2008. Dénommée PLUG : les secrets du musée, elle a été testée le week-end de la Fête de la Science, les 22 et 23 novembre 2008 .

\section{Architecture du jeu}

Lors du dépôt du projet à l'ANR, les partenaires ont défini le but du jeu, son caractère ubiquitaire et son cadre technique. Pour permettre le lien entre les hommes et les objets, il est nécessaire de disposer d'un système de communication sans fil $(\mathrm{WiFi}$, GSM/GPRS/3G+, Radio Fréquence). Le WiFi et le GSM devaient être fournis par le CNAM et l'opérateur partenaire. Pour les radios fréquences, la technologie RFID passive (Radio Fréquence Identification) a été choisie. Des autres éléments constitutifs du jeu (scénario, règles, contenu pédagogique), rien n'avait été spécifié. C'est donc le premier objectif que s'est assigné le consortium, formé par l'ensemble des partenaires.

\section{Prémices}

Plusieurs idées concernant le contenu pédagogique ont servi de point de départ à la construction de l'architecture du jeu (ou game design), dont, en premier lieu, la constatation que les nouvelles technologies ont provoqué l'émergence de nouveaux appareils remplaçant les audio-guides traditionnels. Pour permettre une visite en toute autonomie, nombreuses sont les institutions muséales qui ont investi dans des appareils multimédia. Ces nouveaux systèmes, s'ils autorisent des visites plus riches utilisant non seulement l'ouïe mais aussi la vue, n'en demeurent pas moins structurellement identiques aux précédents : la démarche pédagogique est toujours descendante et les informations données sont les mêmes quel que soit le profil du visiteur. L'établissement culturel est détenteur de l'information qu'il transmet au visiteur sans que celui-ci puisse établir un dialogue ou modifier le degré de détail ou l'angle de vue (historique, social, économique, technique, par exemple) de l'information qu'il reçoit. Le visiteur

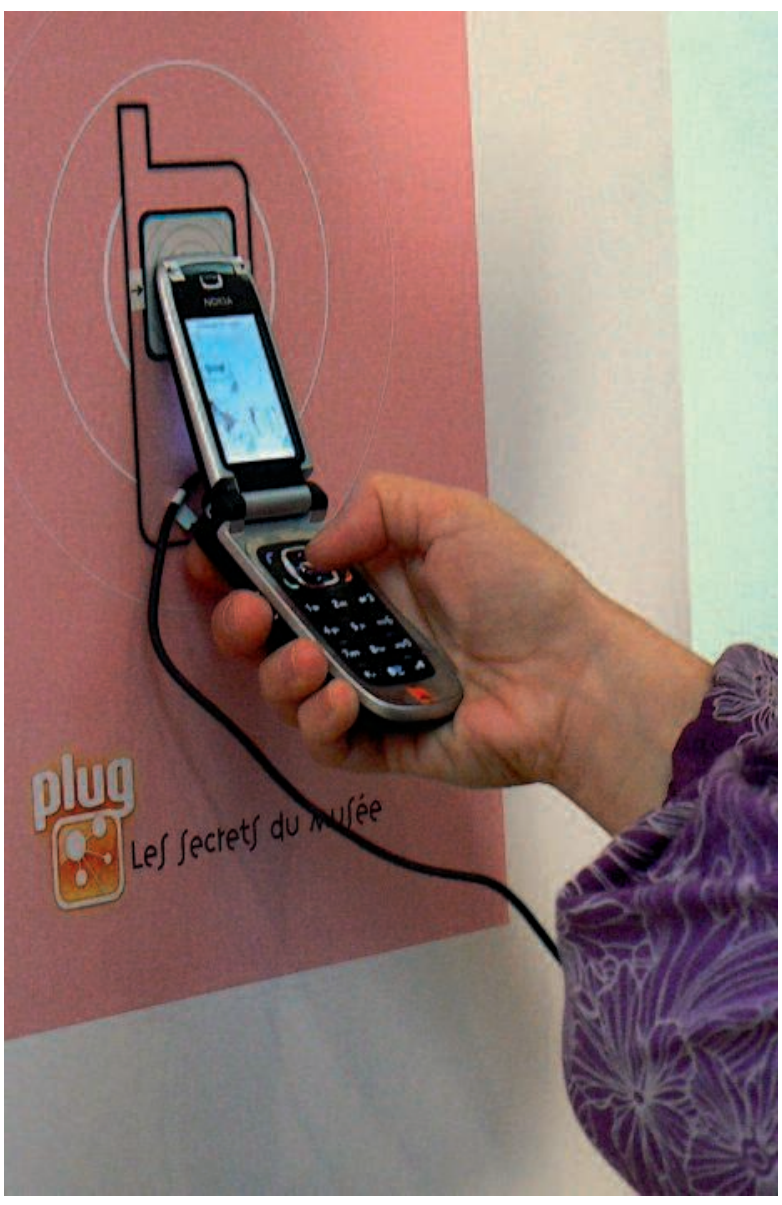

La technologie RFID au service de PLUG () PLUG 
parcourt le musée selon un mode " passif », sans participer, contrairement à ce qu'il pourrait faire lors d'une visite guidée avec un médiateur.

La première mission de PLUG est donc de s'adapter au profil des joueurs, qu'ils puissent jouer selon des stratégies propres à leurs qualités, leurs comportements ou leurs goûts personnels. Mais cela ne doit pas être au détriment de la connaissance de la collection. C'est pourquoi, le jeu doit mettre en valeur quelques objets du musée en poussant le joueur à se renseigner sur ces objets. Cependant, il faut aussi qu'il renvoie vers le contenu disponible au sein du musée pour ancrer le jeu dans le lieu. Ainsi, des éléments de texte de cartels, bornes histoires, tableaux électroniques contenant les vidéos ou des diaporamas, doivent s'intégrer dans le scénario, et inversement, le scénario doit renvoyer vers ces éléments documentaires. Afin que les joueurs puissent élargir leurs faisceaux de sources de renseignements, des liens vers des sites Internet, choisis et référencés par le musée, doivent être mis à leur disposition sur leur terminal mobile. L'utilisation de sources diverses, internes et externes au musée, favorise la dynamique du jeu, en en variant les difficultés et le scénario.

L'accès à des sites externes à l'institution relie cette dernière à d'autres sites d'information scientifique et la met au cœur d'un réseau de connaissance de la culture scientifique et technique. Pour que ce lien entre le joueur et ces sites documentaires, créé au moment du jeu, soit maintenu au-delà de la session de jeu, il nous a paru important que le joueur puisse disposer de ces références en dehors du musée, et, pourquoi pas, continuer à jouer. L'utilisation de la plateforme "Visite + ", développée par la Cité des Sciences, a alors été envisagée. En l'installant sur le site du musée et en y ouvrant un espace spécifique pour PLUG : les secrets du musée, le joueur pourrait s'y connecter après sa visite et la prolonger en y retrouvant les sites, et autres éléments du jeu (quiz, zoom sur les objets vus), ou tout autre jeu à caractère pédagogique, en relation avec l'univers de PLUG.

Cependant, la technique nous a obligés à renoncer à certaines de nos idées de départ. En effet, très vite, il est apparu que l'installation du WiFi au sein du musée ne serait pas possible pour l'échéance de la Fête de la Science et que les forfaits data pour les téléphones mobiles risquaient de ne pas être disponibles. Il a fallu trouver une solution au cas où ils feraient défaut entraînant une architecture technique de jeu réduite. La technologie RFID devenait la seule disponible de façon certaine et donc la construction du jeu PLUG : les secrets du musée s'est organisée autour de ces principes. La proposition d'accès vers des sites externes, l'utilisation de «Visite + » et l'idée de connexion des objets entre eux ont été abandonnées. Toutes les informations nécessaires au joueur devaient être embarquées sur le terminal ou intégrées dans l'étiquette RFID qui a pris la forme d'une étiquette autocollante semblable à celles collées au revers de certains livres et faisant fonction d'antivol. Dotée d'une mémoire de $1 \mathrm{Ko}$, chacune pouvait emmagasiner des données susceptibles d'être lues, modifiées, voire effacées par un équipement lecteur, un téléphone portable-lecteur RFID, disponible dans le commerce. Ce terminal mobile est également le support de jeu.

\section{Idées préalables à la mise en place}

Ces choix techniques permettaient tout de même de satisfaire aux contraintes imposées par le musée. En premier lieu, le jeu devait être discret, c'est-à-dire que les visiteurs-joueurs ne devaient pas gêner les «non joueurs ». Le musée souhaitait aussi que le jeu soit à destination de tous les publics, enfants, adolescents, jeunes et moins jeunes, seuls ou en famille. Il devait donc s'adapter au profil du joueur et apporter à chacun un contenu pédagogique adéquat.

Il fallait également que le joueur puisse découvrir l'ensemble du musée de façon totalement autonome. En effet, un des objectifs de PLUG : les secrets du musée était de compléter les offres de visites guidées générales ou thématiques proposées par ailleurs par le musée autour des sept domaines exposés : "Instruments scientifiques », « Matériaux »,

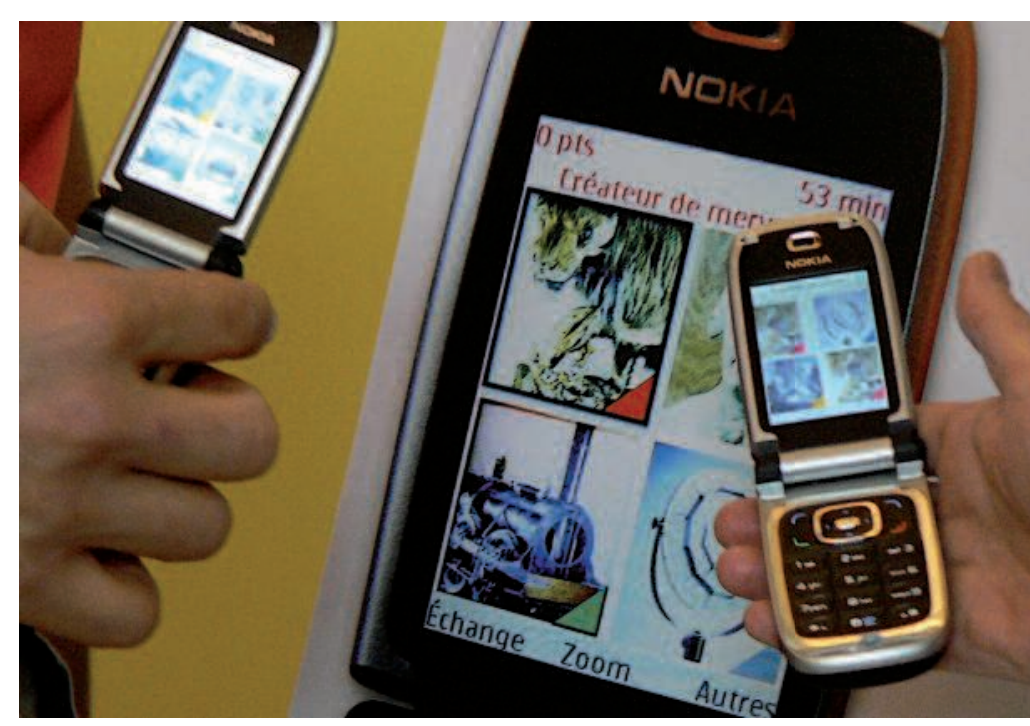

L'écran du téléphone :

le support de jeu indispensable des joueurs (c) PLUG 
« Construction », «Communication », «Énergie », « Mécanique », «Transport». Le jeu avait pour objectif de montrer d'autres objets, d'une façon transversale, ne reprenant pas forcément la chronologie de l'exposition permanente. Enfin pour que la solution proposée soit viable d'un point de vue économique, elle se devait d'être peu coûteuse, fiable et demander une maintenance réduite pour une installation permanente dans le musée.

Il fallait cependant garder à l'esprit que PLUG : les secrets du musée est avant tout un jeu. Donner un aspect ludique au projet était donc incontournable pour légitimer l'appellation jeu. Le ressort ludique choisi en conséquence, maintes fois utilisé et dont l'efficience n'est plus à prouver, a donc été le suivant : réunir un maximum de points dans un temps limité.

Il apparaissait aussi important de donner aux joueurs la possibilité de développer différentes stratégies de jeu afin que chacun y trouve son compte : petits, adultes, stratégique, à risque, fonceur, prudent... Une liberté totale était accordée au joueur : jeu d'alliance, jeu solitaire, voire triche, aucun comportement ne lui était imposé. Pour cela, le game design a été conçu pour favoriser les échanges et la communication entre joueurs. Il permettait aussi de créer une émulation particulière durant le jeu. Pour que le joueur puisse jouer à sa manière, l'architecture devait être la plus ouverte possible et proposer plusieurs modes de jeu, en fonction des qualités ou des goûts de chacun. Curiosité, pragmatisme, ouverture d'esprit, compétitivité, civisme ont été les caractéristiques de profils choisis, en clin d'œil au profil idéal du chercheur. En les utilisant comme ressort du jeu, nous mettions en avant les qualités du joueur lui-même, quel qu'il soit. À chaque trait de caractère nous avons fait correspondre un comportement de jeu. Par exemple, l'esprit d'ouverture se traduisait par un échange d'informations entre joueurs, la compétitivité devenait l'envie de gagner le plus de points, la curiosité était la volonté d'apprendre en s'informant sur les objets du musée. À chaque fois qu'une qualité était mise en œuvre, des points étaient donnés au joueur. Tous les joueurs, quels que soient leur âge et leur profil, pouvaient se retrouver dans le jeu.

Les règles du jeu

Les règles du jeu s'inspirent du jeu des sept familles. Seize objets du musée ont été choisis et répartis dans quatre familles différentes. Les objets réels sont chacun représentés par trois cartes virtuelles identiques (ou objets numériques). Les objets numériques ont en effet été triplés pour éviter de bloquer le jeu en cours de partie. L'une des cartes virtuelles est implantée dans une étiquette RFID, collée sur un trépied d'exposition (ou borne), à côté de l'objet réel, le rendant facilement identifiable sur le parcours du jeu. Elle peut être recueillie lors de la lecture de l'étiquette radio fréquence. Les deux autres sont réparties entre les huit téléphones portables qui équipent les joueurs lors de chaque session. La distribution entre les bornes et les terminaux est tout à fait aléatoire et s'effectue au début de chaque partie.

Avant que la partie commence, les joueurs décident de jouer seul ou en équipe. Pour plus de simplicité, nous appellerons " équipe » un joueur isolé ou plusieurs joueurs jouant avec un seul terminal. Chaque équipe reçoit un téléphone mobile et une famille à réunir. Chaque téléphone possède en mémoire quatre cartes différentes appartenant chacune à une famille différente. Les équipes doivent arpenter le musée à la recherche des bornes, pour retrouver les trois autres cartes, toutes différentes, afin de compléter leur

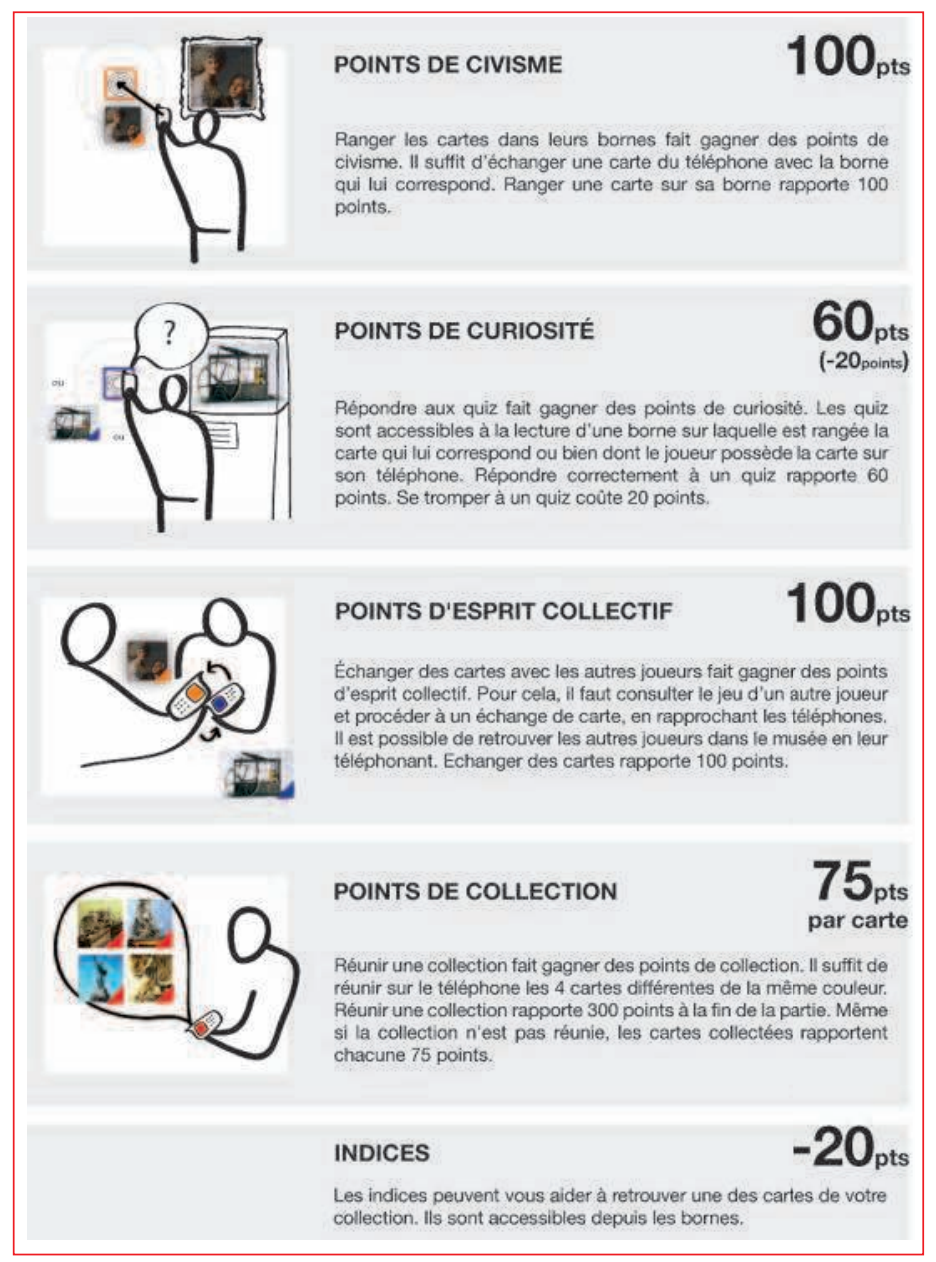

II existe plusieurs manières de gagner des points : tout dépend du profil du joueur. 
famille. Elles peuvent lire le contenu des bornes par l'intermédiaire de leur téléphone grâce à la technologie RFID. Elles décident alors de déposer ou non une carte virtuelle rangée dans leur téléphone en échange de la carte virtuelle rangée dans la borne. C'est la règle de l'échange équivalent : un objet numérique dans la borne contre un objet numérique dans le téléphone, pas plus pas moins. La même règle s'applique lorsque les équipes décident d'échanger des cartes entre elles. Durant la partie, elles peuvent utiliser leur téléphone, comme... un vrai téléphone, pour se contacter, se renseigner sur les cartes des autres équipes et se retrouver à un point de rendez-vous dans le musée - cette règle a d'ailleurs été détournée par certains joueurs qui fixaient des points de rendez-vous non pas pour s'y rendre et échanger des cartes, mais dans le but de faire perdre du temps aux autres équipes! L'échange de cartes entre équipes s'effectue par l'intermédiaire des téléphones. Par l'interface écran, la touche d'échange est sélectionnée puis les deux téléphones sont rapprochés l'un de l'autre jusqu'à ce qu'ils soient en quasi-contact. Le transfert s'effectue alors.

D’autres éléments de scénarisation viennent compléter la mise en contexte des objets. Les textes étant volontairement en quantité modérée dans le musée, des éléments de contenu supplémentaires étaient nécessaires pour le jeu. Des textes accessibles via le téléphone sont donc proposés aux équipes : ce sont les zooms dans lesquels l'objet se raconte lui-même. Les quiz sont également une autre manière d'aborder les objets. Deux des trois réponses proposées pour chaque question du quiz sont ludiques : en plus d'aider les joueurs à trouver la bonne réponse avec un minimum de réflexion, l'aspect ludique a l'avantage de démystifier l'objet en détournant tout le poids de connaissance qui lui est associé. Les répliques participent elles aussi à découvrir l'objet de manière inattendue. Dans PLUG: les secrets $d u$ musée, chaque objet s'exprime oralement (via des écouteurs branchés au téléphone) dès qu'il est déplacé du téléphone à une borne, ou d'une borne au téléphone. Très théâtralisées, les répliques apportent des éléments de contenu rigoureux, même s'ils sont acquis de façon plus inconsciente par le joueur.

La mission des équipes est donc de parcourir le musée et de retrouver les cartes, l'équipe qui a remporté le maximum de points gagne la partie. Le gain de points peut se faire de plusieurs manières possibles, ce qui permet aux équipes de développer différentes stratégies en fonction des situations et de leur profil. Ainsi, dès lors que l'on range les cartes virtuelles dans les bornes près des objets qu'elles représentent, l'équipe gagne 100 points de civisme. En répondant aux quiz, 60 points de curiosité sont attribués pour chaque bonne réponse (mais 20 points sont perdus à chaque mauvaise réponse). Échanger des cartes avec les autres fait gagner 100 points d'esprit collectif. Réunir une famille complète rapporte 300 points (75 points par cartes collectées). Demander un indice pour retrouver les cartes de sa famille coûte 20 points.

\section{Réalisation et expérimentation}

\section{Réalisation}

La mise en place des familles résulte d'un long travail de pédagogie et de scénarisation. Le musée se caractérise par sa très riche collection d'objets. Ces derniers ont été mis en avant tout au long de la mise en place du scénario. Il a fallu choisir seize objets parmi près de trois mille exposés : le processus de sélection a donc été minutieux et longuement discuté. Certains objets phares contribuant à l'identité même du musée, nous semblaient incontournables: le pendule de Foucault, l'avion n³ de Clément Ader ou encore la joueuse de tympanon ont donc été retenus. Mais il nous apparaissait également important de mettre en lumière certains objets méconnus du grand public, ainsi que des inventeurs. Les objets ont ensuite été répartis dans quatre familles différentes, imaginées dans l'idée de surprendre les joueurs-visiteurs. Elles ont été réalisées en se fondant sur des points de vue historique, social et scientifique, tout comme chaque objet se caractérise historiquement, socialement et scientifiquement :

- la famille «Dompteurs d'élément » renvoie à la science d'avant 1783 , époque où elle se base sur les travaux d'Aristote, vieux de 2000 ans. D'après ce dernier, toute chose sur Terre était composée des

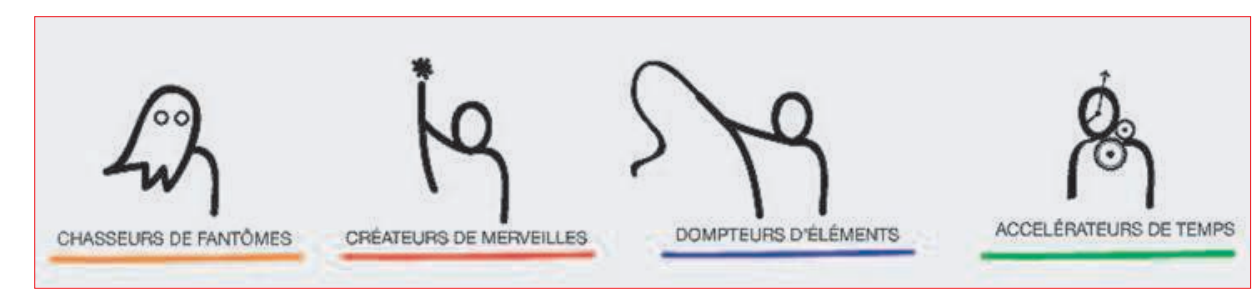

Les quatre familles du jeu 
éléments eau, terre, feu et/ou air, avant que l'expérience de la synthèse de l'eau réalisée en 1783 par Lavoisier démontre par l'expérience l'invalidité de cette théorie. La famille «Dompteurs d'éléments» a été composée pour rappeler la science d'autrefois avec les 4 éléments eau (pont de Bordeaux), terre (tunnelier de Madrid), feu (lentille de Fresnel) et air (l'avion de Clément Ader) ;

- la famille «Chasseurs de fantômes » souhaite rendre hommage aux inventeurs cachés derrière chaque objet. Les grands personnages dont les travaux et/ou l'histoire ont eu un lien avec le musée, ont été sélectionnés. Antoine-Laurent de Lavoisier (dont la femme Marie-Anne se battit pour récupérer l'ensemble des instruments aujourd'hui répertoriés dans la collection du musée), Jacques de Vaucanson (son métier à tisser a été l'un des premiers objets à rentrer dans la collection du musée), les frères Lumière (leur caméra est un objet phare de la collection), Léon Foucault (dont la mère a fait don au musée de l'ensemble des pendules ayant servi à ses diverses expériences) ;

- la famille "Créateurs de merveilles » surprend en répertoriant des objets de façon inattendue, en se basant sur leur aspect "plaisir et divertissement ». La joueuse de tympanon, la machine de Marly, le lion et le serpent ou encore la Statue de la Liberté ont tous été réalisés soit pour répondre à un caprice, soit par pure passion, soit pour rendre hommage ; aucun ne répondait à une commande ou un besoin préalable, même s'ils furent reconnus comme pures merveilles de technologies et de techniques;

- la famille «Accélérateurs d'époque » présente les objets ayant joué un rôle primordial lors de la révolution industrielle du XIXe siècle; que ce soit dans les domaines de la communication, des transports, de la fabrication ou encore de l'habillement, la presse de Marinoni, la locomotive de Stephenson, la machine à vapeur de Watt et la mule-jenny ont chacune contribué au changement et à l'évolution soudaine de la société au XIXe siècle.

Un titre surprenant et évocateur était indispensable pour accrocher les visiteurs et susciter leur curiosité quant au choix des objets. Le titre devait également rendre le joueur-visiteur acteur dans le jeu, ce qui explique que les noms de collection renvoient au rôle même du joueur dans sa quête : dompteur, créateur, chasseur ou accélérateur... à lui d’être! Les mots «éléments », «merveilles », « fantômes » et « époque » ont également été soigneusement choisis afin de traduire au mieux l'identité de chaque collection.

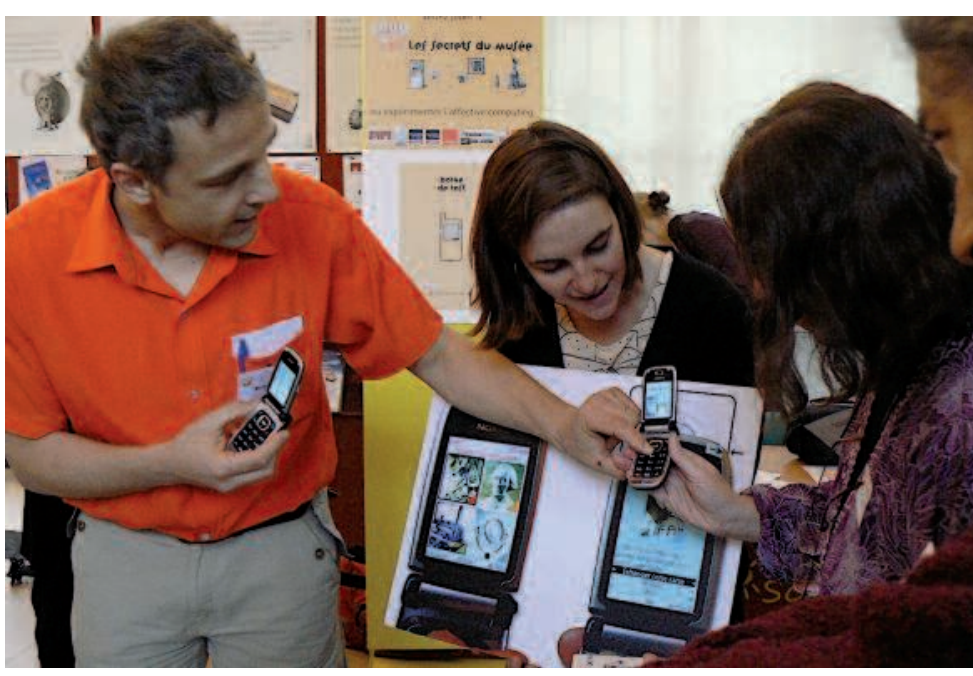

Présentation des règles du jeu aux joueurs lors de la Fête de la Science 2008

() PLUG

Les répliques ont demandé un travail de médiation inédit. En plus de l'immersion des joueurs par l'ouie, les répliques permettent de raconter l'objet autrement : nous nous sommes en effet interrogés sur la personnalité même des objets. S'ils avaient été vivants, auraient-ils été femme ou homme ? Quel aurait été leur caractère en fonction de leur inventeur, du contexte historique dans lequel ils ont baigné ? En fonction de la borne où l'objet est déposé ou en fonction de la famille quili rejoint sur le téléphone, l'objet exprime donc un sentiment de colère, de contentement, de surprise...

\section{Expérimentation}

Avant le déploiement du jeu grandeur nature, des tests ont été réalisés avec l'aide du personnel du musée et les membres de l'équipe du projet. L'équipe sécurité du musée s'est fortement impliquée : le jeu étant un dispositif nouveau, cela lui a permis de se familiariser avec le déplacement et le comportement des joueurs-visiteurs. Pour l'équipe du projet, ces tests ont été une bonne préparation à la gestion de la hotline et aux nombreuses questions susceptibles d'être posées par les joueurs. Les principales difficultés rencontrées ont été les bugs techniques et le comportement des joueurs enclins à courir dans le musée de borne en borne. Ces essais nous ont en outre permis d'améliorer le logiciel sousjacent au jeu, de modifier et d'affiner certaines des règles, comme la répartition des points à gagner. 


\section{Conclusions et perspectives}

L'expérimentation s'est déroulée sur deux jours, par sessions de 50 minutes. Le jeu a passionné tous les publics de 7 et 65 ans. Ce premier prototype du projet PLUG a proposé une visite dynamique du musée, non conventionnelle et dans sa forme et dans son fond. Le jeu a su être discret. En effet, la technologie RFID choisie se limitant à des étiquettes collées sur des trépieds de présentation, permet au jeu de s'intégrer dans la scénographie du musée. Il est également discret dans la perception qu'en ont eue les nonjoueurs dans la mesure où il n'y a jamais eu de rejet des joueurs par les «non-joueurs». Ces derniers ont même été très intrigués par le jeu, certains essayant de lire le contenu des étiquettes RFID avec leur téléphone portable «classique » et s'intéressant plus aux objets situés à proximité des bornes de jeu. D’autre part, l'utilisation de la seule technologie RFID favorise la simplicité du déploiement et de la maintenance du jeu. Il suffit d'installer autant d'étiquettes que d'objets valorisés pour pouvoir jouer. La maintenance se limite à vérifier que les batteries des téléphones sont toujours chargées et que les étiquettes ne se décollent pas et qu'elles sont toujours lisibles.

L'architecture du jeu est intéressante dans la mesure où elle offre la possibilité d'insérer du contenu supplémentaire, voire même une autre approche pédagogique. Par ailleurs, le contenu actuel des quiz, zoom et répliques est une bonne base pédagogique loin d'être à destination unique de PLUG : les secrets du musée. Ce travail de médiation autour des objets peut ainsi être tout à fait réutilisé par l'équipe pédagogique du musée pour d'autres projets.

Ce bilan globalement positif ne doit cependant pas faire oublier que les joueurs ont eu l'impression d'être trop focalisés sur le jeu. L'aspect contenu et pédagogie se laisse dépasser par l'aspect jeu et compétition : le choix de l'objet s'est effectué pour des raisons stratégiques de jeu et non pas sur des choix liés au sens des objets, à leur histoire ou leur technologie. Cela est dû à l'architecture du jeu dans laquelle le décompte de temps pousse les joueurs à se presser pour gagner un maximum de points afin d'être les meilleurs, au détriment de la connaissance des objets : une modification visant à donner plus d'importance à la découverte du musée et de sa collection est donc à envisager. D'autre part, PLUG : les secrets du musée n'a pas bouleversé la démarche pédagogique, qui reste descendante. Les indications données sur les objets étaient identiques pour tous, même si elles jouaient sur plusieurs registres (docte, humoristique). Elles étaient fournies par l'institution et non pas recherchées, collectées voire synthétisées par le joueur.

Pour mettre plus en valeur son contenu pédagogique du jeu, des modifications sont donc à prévoir. Une première solution est de donner plus de points à ceux qui font preuve de curiosité technique et scientifique. Une seconde est de développer le contenu pédagogique afin d'aller encore plus loin dans la découverte de la collection du musée. Peut-être faut-il créer plus de liens entre les objets du musée ? Proposer une observation plus attentive de ceux exposés autour de l'objet du jeu pour mieux comprendre le procédé technique sous-jacent à ce dernier ? PLUG : les secrets du musée proposait un scénario statique. L'adapter au joueur à travers un scénario dynamique, réagissant en fonction du comportement, et pourquoi pas des sentiments du joueur, favoriserait-il l'apprentissage ? Le joueur serait alors complètement immergé, en interaction constante avec le jeu, les objets, le rendant perméable au monde alentours et l'impliquant encore plus dans sa découverte du musée.

Ces questions et ces propositions sont le support de la réflexion sur l'architecture du prochain jeu PLUG qui sera testé en novembre prochain lors de la Fête de la Science.

\section{Note}

(1) http://deptinfo.cnam.fr/xwiki/bin/view/PLUG/ 